\title{
Two mechanisms of ductile fracture: void by void growth versus multiple void interaction
}

\author{
Viggo Tvergaard ${ }^{\mathrm{a}}$, John W. Hutchinson ${ }^{\mathrm{b}, *}$ \\ a Department of Mechanical Engineering, Technical University of Denmark, DK2800 Lyngby, Denmark \\ ${ }^{\mathrm{b}}$ Division of Engineering and Applied Sciences, Harvard University, 29 Oxford Street, Pierce Hall, Cambridge, MA 02138, USA
}

Received 13 February 1002

\begin{abstract}
Two distinct mechanisms of crack initiation and advance by void growth have been identified in the literature on the mechanics of ductile fracture. One is the interaction a single void with the crack tip characterizing initiation and the subsequent void by void advance of the tip. This mechanism is represented by the early model of Rice and Johnson and the subsequent more detailed numerical computations of McMeeking and coworkers on a single void interacting with a crack tip. The second mechanism involves the simultaneous interaction of multiple voids on the plane ahead of the crack tip both during initiation and in subsequent crack growth. This mechanism is revealed by models with an embedded fracture process zone, such as those developed by Tvergaard and Hutchinson. While both mechanisms are based on void nucleation, growth and coalescence, the inferences from them with regard to crack growth initiation and growth are quantitatively different. The present paper provides a formulation and numerical analysis of a twodimensional plane strain model with multiple discrete voids located ahead of a pre-existing crack tip. At initial void volume fractions that are sufficiently low, initiation and growth is approximately represented by the void by void mechanism. At somewhat higher initial void volume fractions, a transition in behavior occurs whereby many voids ahead of the tip grow at comparable rates and their interaction determines initiation toughness and crack growth resistance. The study demonstrates that improvements to be expected in fracture toughness by reducing the population of second phase particles responsible for nucleating voids cannot be understood in terms of trends of one mechanism alone. The transition from one mechanism to the other must be taken into account. () 2002 Elsevier Science Ltd. All rights reserved.
\end{abstract}

Keywords: Fracture toughness; Ductile fracture; Void nucleation and growth

\section{Introduction}

Two distinct mechanisms of crack initiation and advance by void growth have been modeled in the literature on the mechanics of ductile fracture. One view has crack growth initiation as the consequence of a single void interacting with the crack tip at each point along the crack front. Voids more removed from the

\footnotetext{
${ }^{*}$ Corresponding author. Fax: +1-617-495-9837.

E-mail address: hutchinson@husm.harvard.edu (J.W. Hutchinson).
} 
tip are assumed to have little effect on the onset of crack growth. Similarly, subsequent growth is imagined to occur by advance of the tip, void by void, along the crack front. This mechanism is represented by the early model of Rice and Johnson (1970) and the subsequent more detailed finite strain computations of McMeeking (1977), Aravas and McMeeking (1985) and Hom and McMeeking (1989) on the interaction of a crack tip with a single neighboring void. The second view involves the simultaneous growth and interaction of multiple voids on the plane ahead of the crack tip during crack growth initiation and subsequent crack advance. This mechanism emerged from models employing an embedded fracture process zone, such as those explored by Tvergaard and Hutchinson (1992). While both mechanisms are based on void nucleation, growth and coalescence, the inferences from them with regard to crack growth initiation and growth are quantitatively different, particularly with respect to predicted toughness.

Models for each of the mechanisms predict that the fracture toughness as measured by the value of the $J$-integral at initiation of crack growth has the form

$$
J_{\text {Ic }}=C \sigma_{\mathrm{Y}} X_{0}
$$

where $\sigma_{\mathrm{Y}}$ is the initial tensile yield stress and $X_{0}$ is the spacing between voids on the fracture plane or the spacing between the crack tip and the nearest void. Here, $C$ is a numerical coefficient which depends on a number of factors including the strain hardening index, $N$, yield to modulus ratio, $\sigma_{\mathrm{Y}} / E$, initial void volume fraction, $f_{0}$, nucleation strain for voids not present at the start of straining, initial void shape and orientation, among others. For the single void model it will be denoted by $C_{\mathrm{SV}}$, and by $C_{\mathrm{MV}}$ for the multiple void model. The earliest predictions for a single pre-existing spherical void such as those of Rice and Johnson (1970) and McMeeking (1977) give $C_{\mathrm{Sv}}$ ranging from about 2 at $f_{0}=0.01$ to 4 at $f_{0}=0.001$, with a relatively weak dependence on strain hardening and $\sigma_{\mathrm{Y}} / E$. Here, the nominal initial void volume fraction is defined in terms of the initial radius of the void, $R_{0}$, by $f_{0}=\left(2 R_{0} / X_{0}\right)^{3}$. These calculations do not account for the influence of the void on the near-tip stress field, but Aravas and McMeeking (1985) carried out a two-dimensional finite strain computation for the nonlinear interaction of an initial circular cylindrical void ahead of a blunting crack tip. These authors showed that the earlier results significantly overestimate the initiation of crack growth. They obtained $C_{\mathrm{SV}} \cong 1$ in the absence of strain hardening and values with about a $25 \%$ elevation for a strain hardening exponent $N=0.2$. Subsequently, Hom and McMeeking (1989) performed three-dimensional finite strain studies of the growth of a single line of spherical voids periodically spaced along crack front.

By contrast, the model of multiple void interaction of Tvergaard and Hutchinson (1992) for pre-existing spherical voids on the extended crack plane gives $C_{\mathrm{MV}} \cong 0.5$, with a weak dependence on both strain hardening and $f_{0}$. Further discussion of these results will be given in the body of the paper.

The present study will demonstrate that each of the two mechanisms applies to a distinct regime of initial void populations, with the multiple void mechanism applicable at larger void volume fractions and the single void mechanism approached as the initial void volume fraction becomes smaller and smaller. It will be seen that the transition between the two mechanisms takes place within a range of initial void volume fractions representative of many ductile structural alloys. For this reason, the difference between the two models as reflected by the coefficients $C_{\mathrm{MV}}$ or $C_{\mathrm{SV}}$ has practical consequences for understanding the connection between processing improvements and toughness increases in metal alloys. By (1), each model indicates that toughness improvements can be achieved by increasing the spacing between voids (or between the void nucleating particles). Each model also predicts that a slight improvement in toughness can be expected by decreasing $f_{0}$ with $X_{0}$ held fixed. However, this effect is not nearly as large as can be expected when $f_{0}$ falls within the transition between the two mechanism regimes. Then, decreasing $f_{0}$ results in $C$ increasing from $C_{\mathrm{MV}}$ towards $C_{\mathrm{SV}}$, corresponding to a larger increase in $J_{\mathrm{Ic}}$ than can be expected from either mechanism alone.

The geometry of the two-dimensional plane strain model employed in this study is shown in Fig. 1. It is the multiple void version of the Aravas and McMeeking (1985) model. All voids are pre-existing. Ap- 


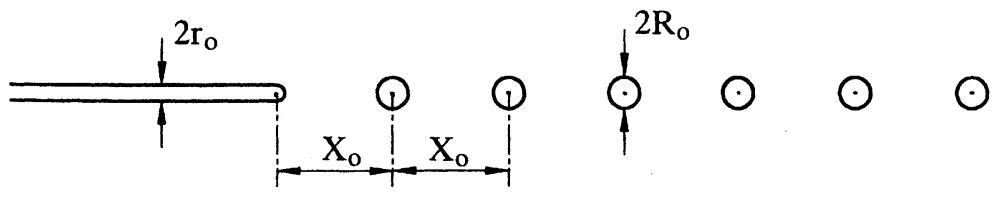

Fig. 1. Geometry of the two-dimensional, plane strain small scale yielding model.

preciable resistance of voids to nucleation will give rise to higher toughness and crack growth resistance than predicted by the present model. It has discrete, initially circular cylindrical voids arranged ahead of the crack tip on the crack plane. Small scale yielding is invoked such that crack is taken to be semi-infinite and loaded remotely by a mode I elastic crack tip field specified by the applied stress intensity factor, $K_{\mathrm{I}}$. The associated applied, or remote, value of the $J$-integral is $J=\left(1-v^{2}\right) K_{\mathrm{I}}^{2} / E$, where $E$ is Young's modulus and $v$ is Poisson's ratio. The material is characterized by isotropic hardening with an initial tensile yield stress, $\sigma_{\mathrm{Y}}$, and strain hardening exponent, $N$. A finite strain, finite element formulation is used. A fine mesh surrounds the crack tip and each void such that the growth of each void is accurately represented. Details of the numerical model are described in Section 2. A criterion for severing the load transmitted by the ligament connecting the current crack tip and void just ahead of it will also be introduced. The two parameters characterizing the initial void geometry are $X_{0}$ and $R_{0}$ (see Fig. 1), or, equivalently, $X_{0}$ and a measure of the initial void volume fraction ahead of the crack defined as $f_{0}=\pi\left(R_{0} / X_{0}\right)^{2}$ for the two-dimensional geometry. The role of the initial radius of the crack tip, $r_{0}$, will also be studied. The model is used to compute the history of $J$ as the crack advances through the array of voids. Definitions of $J_{\text {Ic }}$ will be discussed, including those consistent with current toughness testing standards. Trends of $J_{\text {Ic }}$ as a function of $X_{0}, f_{0}$ and $r_{0}$ will be computed. In addition, solutions will be displayed and inspected at different values of $f_{0}$ with the purpose of identifying which of the two growth mechanisms best describes the behavior.

The approach adopted here is based on model capable of providing high resolution of the growth and interactions of voids ahead of the crack tip, albeit for an idealized arrangement of two-dimensional voids. An alternative possibility for conducting the present investigation would have been to make use of the highly effective computational models of ductile fracture developed over the past decade which employ void-containing elements based on constitutive models such as that of Gurson (1977). There is now an extensive literature on these models represented by papers of Needleman and Tvergaard (1987), Rousselier (1987), Brocks et al. (1995) and Gao et al. (1998). While these models do not resolve the growth of individual voids with the accuracy of the present approach, they should be capable of elucidating the two mechanisms referred to above. To our knowledge, this task has not yet been undertaken by any of the groups involved in developing and implementing the computational models of ductile fracture based on void containing elements. The thrust of the efforts of these groups so far has been devoted to calibration of the models for specific structural alloys and application to geometries and loadings of practical interest. This class of models does not appear to have been used to explore fundamental connections between microstructure and toughness. Nevertheless, a close inspection of results for toughness presented in the literature on these models does reveal some evidence of the trend alluded to above wherein $J_{\mathrm{Ic}} /\left(\sigma_{\mathrm{Y}} X_{0}\right)$ increases much more rapidly with decreasing initial void volume fraction than either of the two mechanisms acting alone would imply.

\section{Numerical model}

The numerical crack calculations carried out here have focus on the growth of voids on the plane in front of the initial crack tip and on the interaction of these voids with crack tip blunting. The analyses are carried 
out for conditions of small scale yielding, under plane strain mode I loading. The elastic-plastic material is described in terms of $J_{2}$-flow theory with isotropic hardening, and the voids are discretely modeled as a number of pre-existing holes in front of the crack. A number of earlier investigations have studied the interaction of a blunting crack tip with one nearby void. The special feature of the present analysis is that several voids in front of the crack are modeled, so that also the interaction between neighboring voids is described during the coalescence process leading to crack growth.

The initial geometry of the solid near the crack tip is illustrated in Fig. 1. Six voids on the crack plane are modeled as circular cylindrical holes with the initial radius $R_{0}$ and spacing $X_{0}$. The crack tip is taken to have a small initial radius $r_{0}$, to facilitate the numerical modeling of blunting, and also the spacing of the tip and the nearest void is taken to be $X_{0}$. Far away from the crack tip, the region analyzed is terminated by an outer circular boundary with radius $A_{0}$. On this outer boundary displacements are specified according to the $K$-field around the initial crack tip, such that loading is applied by incrementally increasing the amplitude $K$ of the edge displacements. Due to symmetry of the solution about the crack plane only half of the region, above the crack plane, is analyzed numerically.

Finite strains are accounted for in the analysis, using a convected coordinate, Lagrangian formulation of the field equations, in which $g_{i j}$ and $G_{i j}$ are metric tensors in the reference configuration and the current configuration, respectively, with determinants $g$ and $G$, and with $\eta_{i j}=(1 / 2)\left(G_{i j}-g_{i j}\right)$ as the Lagrangian strain tensor. The contravariant components, $\tau^{i j}$, of the Kirchhoff stress tensor on the current base vectors are related to the components of the Cauchy stress tensor, $\sigma^{i j}$, by $\tau^{i j}=\sqrt{G / g} \sigma^{i j}$. Then, in the finite strain generalization of $J_{2}$-flow theory, the incremental stress-strain relation is of the form $\dot{\tau}^{i j}=L^{i j k l} \dot{\eta}_{i j}$ with the tensor of instantaneous moduli given by

$$
\begin{aligned}
L^{i j k l}= & \frac{E}{1+v}\left[\frac{1}{2}\left(G^{i k} G^{j l}+G^{i l} G^{j k}\right)+\frac{v}{1-2 v} G^{i j} G^{k l}-\frac{3}{2} \beta \frac{E / E_{\mathrm{t}}-1}{E / E_{\mathrm{t}}-(1-2 v) / 3} \frac{s^{i j} s^{k l}}{\sigma_{\mathrm{e}}^{2}}\right] \\
& -\frac{1}{2}\left(G^{i k} \tau^{j l}+G^{j k} \tau^{i l}+G^{i l} \tau^{j k}+G^{j l} \tau^{i k}\right)
\end{aligned}
$$

Here, the effective Mises stress is $\sigma_{\mathrm{e}}=\left(3 s_{i j} s^{i j} / 2\right)^{1 / 2}, s^{i j}$ is the stress deviator, and the value of $\beta$ is 1 or 0 for plastic yielding or elastic unloading, respectively. The true stress-logarithmic strain curve in uniaxial tension is specified by the power law

$$
\varepsilon= \begin{cases}\sigma / E, & \sigma \leqslant \sigma_{\mathrm{Y}} \\ \left(\sigma_{\mathrm{Y}} / E\right)\left(\sigma / \sigma_{\mathrm{Y}}\right)^{1 / N}, & \sigma \geqslant \sigma_{\mathrm{Y}}\end{cases}
$$

where $E$ is Young's modulus, $\sigma_{\mathrm{Y}}$ is the initial yield stress, and $N$ is the power hardening exponent. In (2), the tangent modulus $E_{\mathrm{t}}$ is the slope of the uniaxial stress-strain curve (3) at the stress level $\sigma_{\mathrm{e}}$.

Approximate solutions for the stress and strain states in the solid are obtained by a linear incremental method, based on using a finite element approximation of the displacement fields in the incremental version of the principle of virtual work. The elements used are quadrilaterals each built-up of four triangular, linear-displacement elements. Examples of the meshes used in the near crack tip region are shown (see ahead to Figs. 5a and 6a) for two different values of the ratio $R_{0} / X_{0}$. High mesh refinement is in place around each void and around the initial crack tip. In fact, this refinement is absolutely necessary to enable the computation of the large void growth and crack tip blunting that occur in some of the cases analyzed. It is noted that the program used to generate the mesh will automatically account for any number of voids specified in front of the crack tip. For the present computations six voids in front of the tip has been considered sufficient.

The value of the $J$-integral is calculated on several contours to check agreement with the prescribed amplitude $K$ of the edge displacements. In the finite-strain context the expression for $J$ is (Rice, 1968; Eshelby, 1970) 


$$
J=\int_{\Gamma}\left[W \mathrm{~d} x^{2}-T^{i} u_{i, 1} \mathrm{~d} s\right], \quad W=\int_{0}^{\eta_{i j}} \tau^{i j} \mathrm{~d} \eta_{i j}
$$

where $\Gamma$ is a path in the reference configuration, from the lower crack surface to the upper crack surface, $\mathrm{d} s$ is an arc length along this path, and $T^{i}$ are the nominal tractions on the boundary of the region enclosed by the contour $\Gamma$. In the present computations the radius $A_{0}$ of the outer boundary has been specified such that it is much larger than the maximum plastic zone size, and in all cases analyzed very good agreement has been found between the prescribed $K$ value and the $J$ values found on several outer contours.

In the present crack growth analyses, growth is analyzed by describing coalescence of neighboring voids or coalescence of the first void with the blunting crack tip. In this way it is possible to predict the crack growth resistance curve. However, to fully describe coalescence by ligaments necking down to zero thickness several re-meshings would be necessary, as has been carried out by Tvergaard (1997) in a cell model study for a single void. In the present analysis a simplified procedure has been adopted, by simply assuming that coalescence occurs when the ligament thickness has been reduced to a ratio $\chi_{\mathrm{c}}$ times the initial ligament thickness. In some analyses the critical value $\chi_{c}=0.33$ or 0.5 is used, while in a few analyses the slightly larger value $\chi_{\mathrm{c}}=0.6$ has been used to in order to avoid numerical break down due to strong mesh distortion. The criterion in the original studies by Rice and Johnson (1970) and McMeeking (1977) assumed the ligament failed when its length dropped to one half the deformed width of the void, corresponding approximately to $\chi_{\mathrm{c}}=0.5$ by the present criterion. When the critical value $\chi_{\mathrm{c}}$ has been reached in a ligament, the requirement of zero normal displacement on the symmetry line at the center of the ligament is dropped, and the corresponding nodal forces on this symmetry plane of the ligament are stepped down to zero over several subsequent increments. The number of increments used to step down these nodal forces is important for the numerical stability of the solution, and in the computations to be presented the number of increments used for this step down process is usually 800 or up to several times that number.

\section{Initiation of crack growth}

Practical criteria for crack growth initiation, such as the one proposed by ASTM (1997) for toughness testing of ductile structural alloys, typically require the identification of a critical crack tip loading parameter after a small amount of crack advance. In this section, we begin by adopting the criterion that has been widely used in theoretical modeling of the initiation of crack growth by assuming that initiation occurs with failure of the ligament between the tip and the nearest void, as detailed in Section 2. In effect, this implies that initiation occurs after a crack advance of approximately the void spacing, $X_{0}$. Sensitivity of predictions to the extent of crack advance at initiation will be addressed in Section 4 .

With the adoption of the criterion based on failure of the first ligament, the computational model described in Section 2 gives rise to predictions in the form of (1), where dimensional analysis implies that the dimensionless coefficient in (1) is a function of the following combination of parameters:

$$
C=F\left(f_{0}, \frac{r_{0}}{X_{0}}, \frac{\sigma_{\mathrm{Y}}}{E}, N, v\right)
$$

Recall that the initial volume fraction of the voids on the plane ahead of the tip, $f_{0}$, has been defined in terms of the initial geometry of the plane strain model in Fig. 1 as $f_{0}=\pi\left(R_{0} / X_{0}\right)^{2}$, and the initial radius of the crack tip is $r_{0}$. In this paper, emphasis will be placed on the roles of $f_{0}$ and $r_{0} / X_{0}$. There is a relatively weak dependence on the value of $\chi_{\mathrm{c}}$, characterizing the point at which the ligaments fail, which will also be demonstrated. The influences of the other dimensionless parameters, $\sigma_{\mathrm{Y}} / E, N$ and $v$, are similar for each of the two basic models and will not be investigated here. For all the results presented in this paper, 


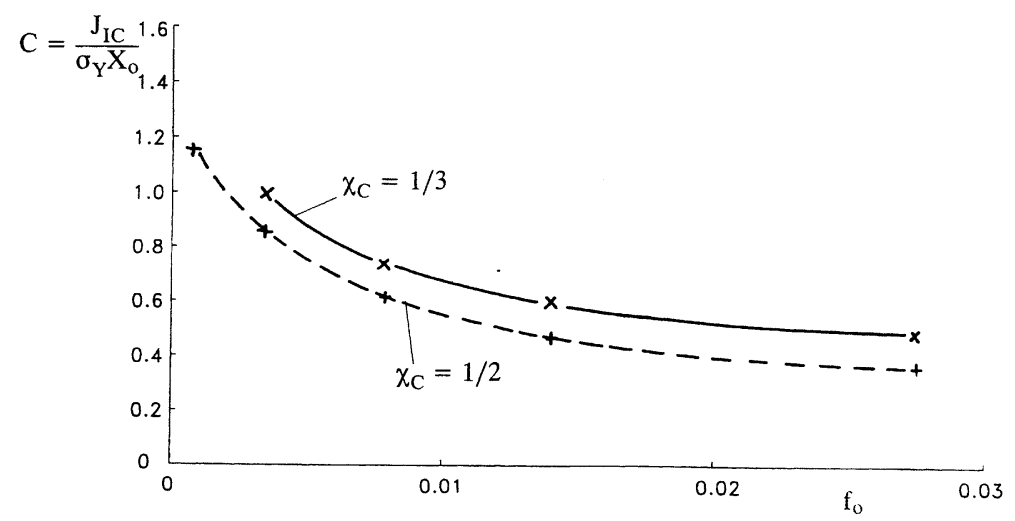

Fig. 2. Model results for $C=J_{\mathrm{Ic}} /\left(\sigma_{\mathrm{Y}} X_{0}\right)$ as a function of $f_{0}=\pi\left(R_{0} / X_{0}\right)^{2}$. All results for $N=0.1, \sigma_{\mathrm{Y}} / E=0.003$ and $v=0.3$. Results are determined with either $\chi_{\mathrm{c}}=0.2$ or 0.3 and with $r_{0} / X_{0}=1 / 10,1 / 15$ and $1 / 30$, as indicated.

$$
\sigma_{\mathrm{Y}} / E=0.003, \quad N=0.1, \quad v=0.3
$$

Computed values of $C \equiv J_{\text {Ic }} / \sigma_{\mathrm{Y}} X_{0}$ are displayed against the initial void volume fraction, $f_{0}$, in Fig. 2 for values of $f_{0}$ ranging from smaller than 0.001 to almost 0.03 . As $f_{0}$ decreases over this range, there is a clear trend for $C$ to increase from values representative of the multiple void model, $C_{\mathrm{MV}}$, toward values representative of the single void model, $C_{\mathrm{SV}}$, as determined by Aravas and McMeeking (1985). The range of initial void volume fractions in Fig. 2 falls within the transition between the two mechanisms. The two to threefold increase of $C$ with decreasing $f_{0}$ is considerably greater than any prediction from either model by itself, excluding the earliest single void models which do not account for the influence of the void on the near-tip field. The range of initial void volume fractions in Fig. 2 spans a wide class of material systems of practical interest, although representative values $f_{0}$ for some of the toughest alloys could be even smaller than 0.001 .

The effect of the ligament reduction, $\chi_{c}$, at ligament failure is also reflected in Fig. 2 for results computed with $\chi_{\mathrm{c}}=1 / 2$ and $1 / 3$. For the smaller of the two values of $\chi_{\mathrm{c}}, C$ is approximately 0.1 larger than for the larger $\chi_{\mathrm{c}}$ for all values of $f_{0}$. Thus, the effect of this parameter is not large. It does, however, have a fairly strong impact on the difficulty of the numerical calculations. The smaller is $\chi_{\mathrm{c}}$, the greater is the distortion of elements in the ligaments at the point of failure, with corresponding increases in computational time and convergence difficulty. The computation at the smallest value considered, $f_{0}=0.000873$, with $\chi_{\mathrm{c}}=1 / 3$ would have required re-meshing, which has not been employed. Most of the results in Fig. 2 have been computed with an initial crack tip radius given by $r_{0} / X_{0}=0.1$. There is almost no effect if smaller values of initial tip radius are chosen. This has been verified by additional computations with $r_{0} / X_{0}=1 / 15$ and $1 / 30$ for $f_{0}=0.0035$. As long as $r_{0} / X_{0}$ does not exceed about 0.1 and as long as $\chi_{\mathrm{c}}$ is less than about $1 / 2, C$ depends primarily on $f_{0}$, with some dependence on $N$ which is not determined in this paper.

Dramatic evidence for the distinct behavioral mechanisms is revealed by the void growth plots of Fig. 3. The ratio of the area of the void to its initial area, $A / A_{0}\left(A_{0}=\pi R_{0}^{2}\right)$, is plotted as a function of crack tip loading, $J / \sigma_{\mathrm{Y}} X_{0}$, for each void ahead of the crack tip with numbering starting with 1 for the closest void, 2 for the next closest, etc. All the growth curves pertain to growth without any failed ligaments. At the smallest initial void volume fraction, $f_{0}=0.00087$, only the closest void undergoes any significant growth, reaching about 40 times its initial area before failure of the first ligament. This is indicative of the single void mechanism. By contrast, the behavior displayed in Fig. 3 for the largest of the three values of $f_{0}$, shows that many voids ahead of the tip are growing simultaneously with very little difference in the rate of growth for the three closest voids to the tip. In fact, there is a range of $J / \sigma_{\mathrm{Y}} X_{0}$ when the second void grows slightly 


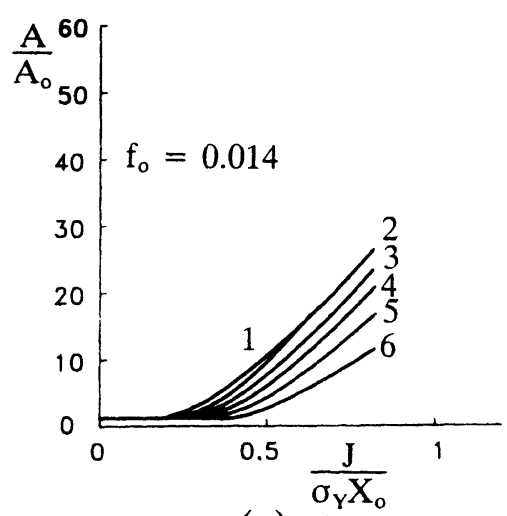

(a)

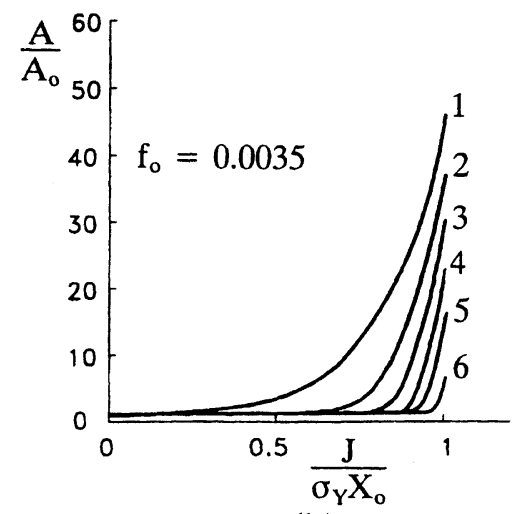

(b)

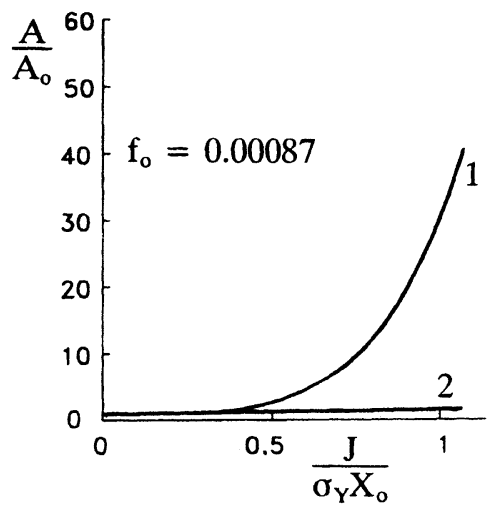

(c)

Fig. 3. Model predictions for growth of individual voids ahead of the tip prior to failure of the first ligament as a function of remote loading for three initial void volume fractions. The voids are numbered in relation to their position from the crack tip with \#1 as the closest. The current cross-sectional area of a void is $A$ and its initial value is $A_{0}$. The smallest $f_{0}$ lies nearly within the regime of the single void mechanism while the largest $f_{0}$ falls within the regime of the multiple void mechanism. All results for $N=0.1$, $\sigma_{\mathrm{Y}} / E=0.003, v=0.3$ and $r_{0} / X_{0}=0.1$.

faster than the first. This case illustrates the multiple void mechanism. The void growth curves shown in Fig. 3 for the intermediate value of $f_{0}$ illustrates a case clearly in the transition between the single and multiple void mechanisms. The void closest to the tip experiences the greatest growth, but appreciable growth of the voids further away from the tip occurs as well. Indeed, it can be seen that the rate of growth of the more distant voids exceeds that of the closest void at the larger values of crack tip loading.

This same data on computed void growth rates is plotted in a different manner in Fig. 4. Here, $A / A_{0}$ is plotted against void position ahead of the tip at several levels of $J / \sigma_{\mathrm{Y}} X_{0}$, again in each case for loading prior to any ligament failures. The behavior described in connection with Fig. 3 is again evident. Very little growth of other than the first void takes place for the material with the smallest value of $f_{0}$, while

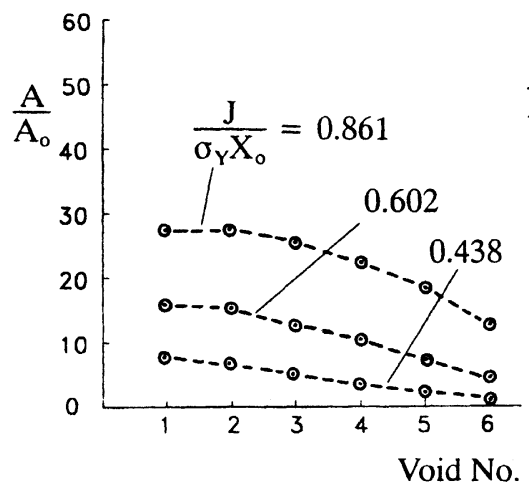

(a)

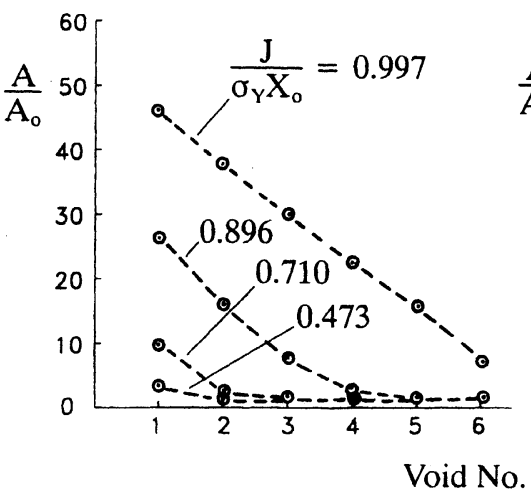

(b)

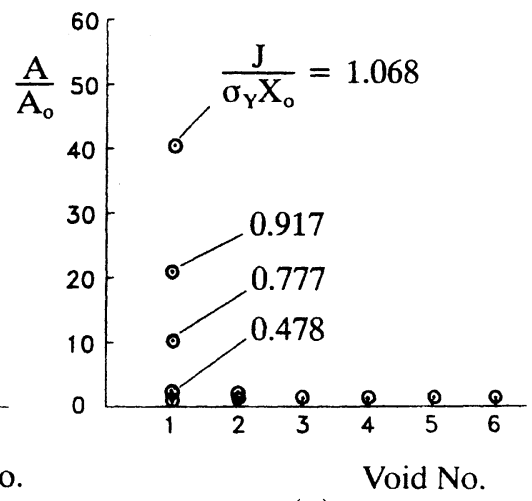

(c)

Fig. 4. Model predictions for growth of individual voids ahead of the tip prior to failure of the first ligament at various levels of remote loading for three initial void volume fractions. These are the same results plotted in Fig. 3, but here as a function of position ahead of the crack tip. 


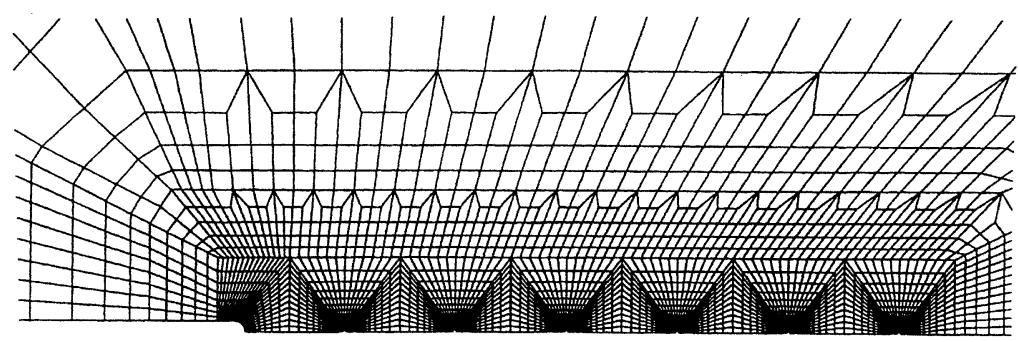

(a)

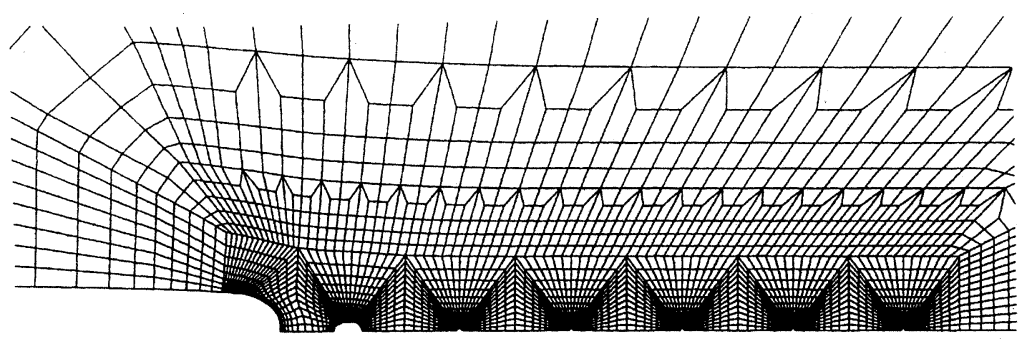

(b)

Fig. 5. (a) Initial mesh and (b) deformed mesh at the point where the first ligament has been reduced to $1 / 2$ its original width for $f_{0}=0.00087$ corresponding to an initial void volume fraction within the regime of the single void mechanism $\left(N=0.1, \sigma_{\mathrm{Y}} / E=0.003\right.$, $v=0.3$ and $r_{0} / X_{0}=0.1$.

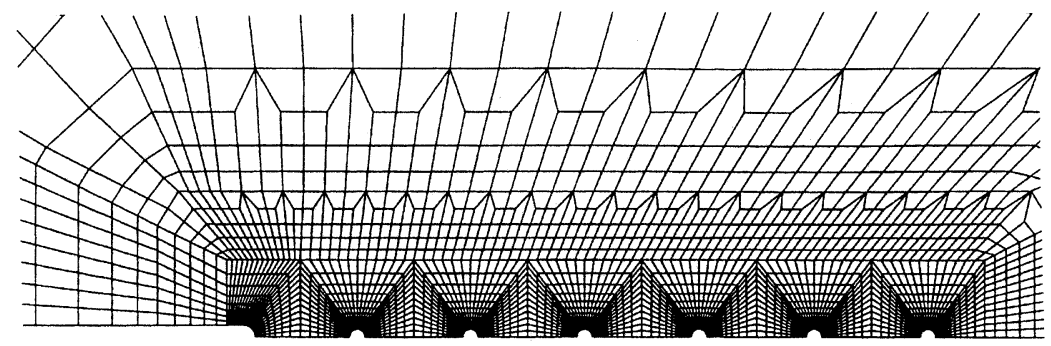

(a)

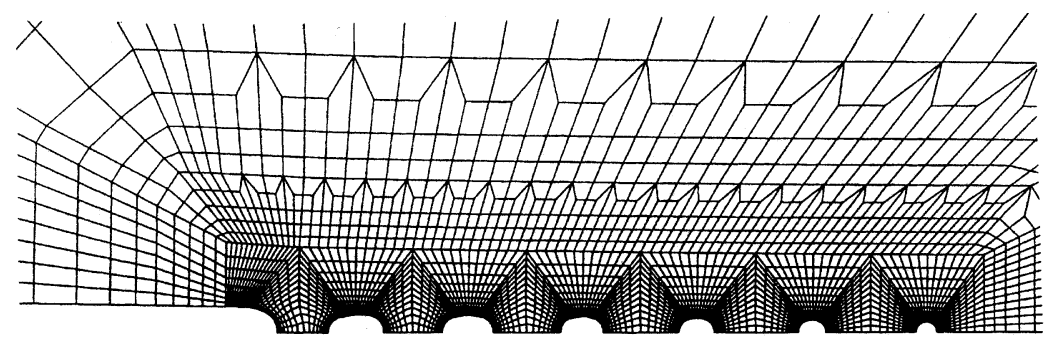

(b)

Fig. 6. (a) Initial mesh and (b) deformed mesh at the point where the first ligament has been reduced to $1 / 2$ its original width for $f_{0}=0.014$ corresponding to an initial void volume fraction within the regime of the multiple void mechanism $\left(N=0.1, \sigma_{\mathrm{Y}} / E=0.003\right.$, $v=0.3$ and $\left.r_{0} / X_{0}=0.1\right)$. 
appreciable growth of all six voids ahead of the tip occurs for the case of the largest value of $f_{0}$. The results for $f_{0}=0.0035$ clearly illustrate transitional behavior, as already noted.

The computational finite element meshes in the initial geometry and in the deformed configuration just prior to failure of the first ligament are equally revealing. Fig. 5a, shows the portion of the initial mesh that contains the crack tip and the six voids ahead of the tip for the case $f_{0}=0.00087$. The deformed state of this mesh at the point where the ligament has been reduced to about $1 / 2$ of its initial value is seen in Fig. $5 \mathrm{~b}$. The predominance of the growth of the first void is evident. The corresponding meshes for the case with $f_{0}=0.014$ are shown in Fig. $6 \mathrm{a}$ and $\mathrm{b}$, revealing the tendency for all the voids near the tip to experience growth. One feature of the growth of the voids that can be seen in both cases is their tendency to become oblate, as is expected from basic results for growth of voids at high stress triaxiality (Budiansky et al., 1982), and as noted also by Aravas and McMeeking (1985).

\section{Crack advance}

The calculations reported for crack growth initiation were continued past the failure of the first ligament to allow the crack tip to advance to the next ligament, fail it, etc. As discussed in Section 2, the nodal forces transmitted by the elements in a given ligament are reduced to zero when the ratio of the deformed crosssectional width of the ligament to the initial width reaches $\chi_{\mathrm{c}}$. Advance, $\Delta a$, takes place in discrete increments of length $X_{0}$. Between failure of one ligament and the next, the change in $J$ is computed. In this way, the history of $J$ versus $\Delta a$ (i.e. the crack growth resistance, $J_{\mathrm{R}}(\Delta a)$ ) can be obtained using the model introduced in Section 2. Computed resistance curves for seven values of $f_{0}$ are presented in Fig. 7. For each

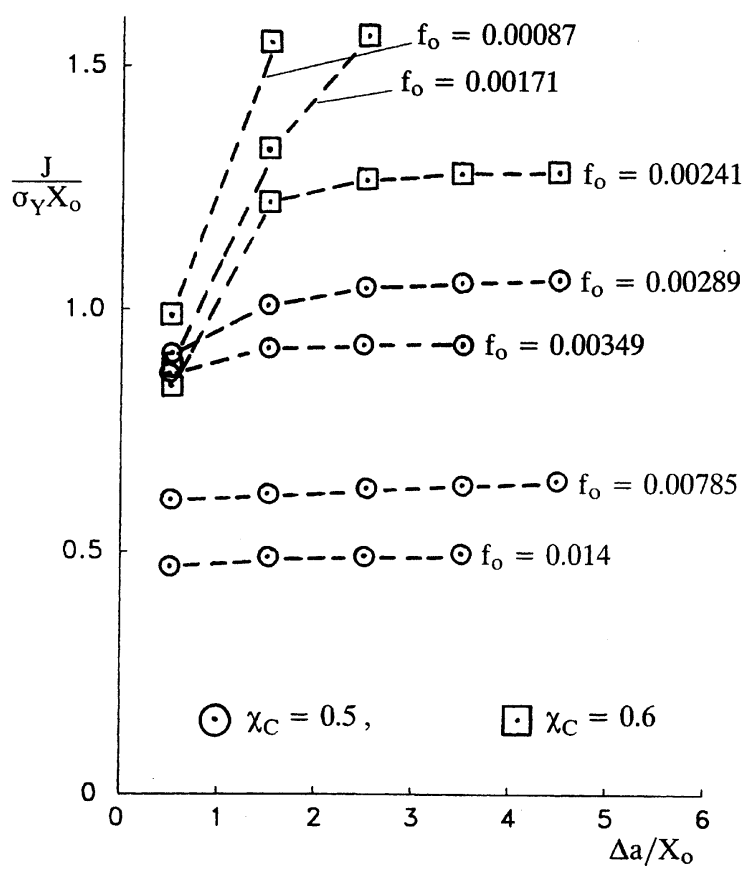

Fig. 7. Crack growth resistance curves for various $f_{0}$ as predicted by the two-dimensional discrete void model in each case for $N=0.1$, $\sigma_{\mathrm{Y}} / E=0.003, v=0.3$ and $r_{0} / X_{0}=0.1$. The point corresponding to failure of the first ligament is plotted at the normalized crack advance, $\Delta a / X_{0}=1 / 2$, to failure of the second ligament at $\Delta a / X_{0}=3 / 2$, etc. For the three smallest $f_{0}$ the criterion for failure of the ligaments was specified by $\chi_{\mathrm{c}}=0.6$, while $\chi_{\mathrm{c}}=0.5$ for all the others. 
curve, the value of $J$ at initiation corresponds to failure of the first ligament, as before, and is plotted at $\Delta a / X_{0}=1 / 2$. The value of $J$ at the failure point of the second ligament is plotted at $\Delta a / X_{0}=3 / 2$, and so forth.

The resistance curves for the two largest values of $f_{0}$ display very little increase in $J$ for growth out to four voids from the initial position of the tip. For $f_{0}=0.014$, Fig. 8a shows the portion of the mesh surrounding the initial tip and all the voids at the point just after the fourth ligament has been released. By comparing this plot with the corresponding one in Fig. $6 \mathrm{~b}$ for the same system at the point of failure of the first ligament, it can be seen that relatively small additional void growth is required to fail four ligaments. Accordingly, the crack growth resistance curve is almost flat. Crack growth resistance becomes substantial at smaller values of $f_{0}$. At the smallest value considered here, $f_{0}=0.00087, J$ increases by more than $50 \%$ by advancing one void spacing. The mesh just after failure of the second ligament in this case is shown in Fig. $8 \mathrm{~b}$, where it can clearly be seen that the mechanism is void by void growth. (In the interest of reducing computational difficulty, the criterion for failure of the ligaments was taken to be $\chi_{c}=0.6$ in the results in Figs. 7 and 8 for the three smallest initial void volume fractions while $\chi_{c}=0.5$ for the others.) The calculations for two intermediate initial void volume fractions, $f_{0}=0.0029$ and 0.0024 , in Fig. 7 reveals that initiation appeared to be represented by the single void mechanism. However, as $J$ increases and the crack advances, voids beyond the one closest to the tip begin to experience significant growth and the behavior becomes increasingly similar to that of the multiple void mechanism.

Define $S$ as the slope following initiation of the dimensionless resistance curve in Fig. 7, i.e.

$$
S=\frac{\mathrm{d}\left(J / \sigma_{\mathrm{Y}} X_{0}\right)}{\mathrm{d}\left(a / X_{0}\right)}=\frac{1}{\sigma_{\mathrm{Y}}} \frac{\mathrm{d} J}{\mathrm{~d} a}
$$

For the smallest initial void volume fraction, $f_{0}=0.00087$, this slope is $\approx 0.5$, corresponding to experimentally measured slopes of some of the toughest structural alloys. Thus, we believe that the smallest initial

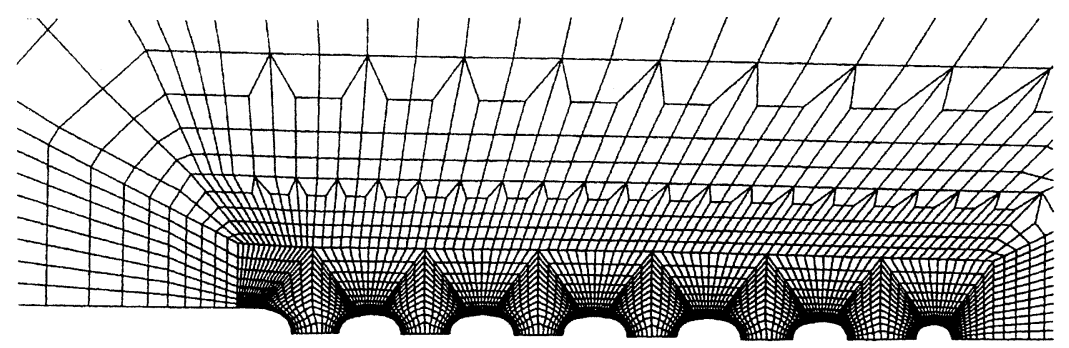

(a)

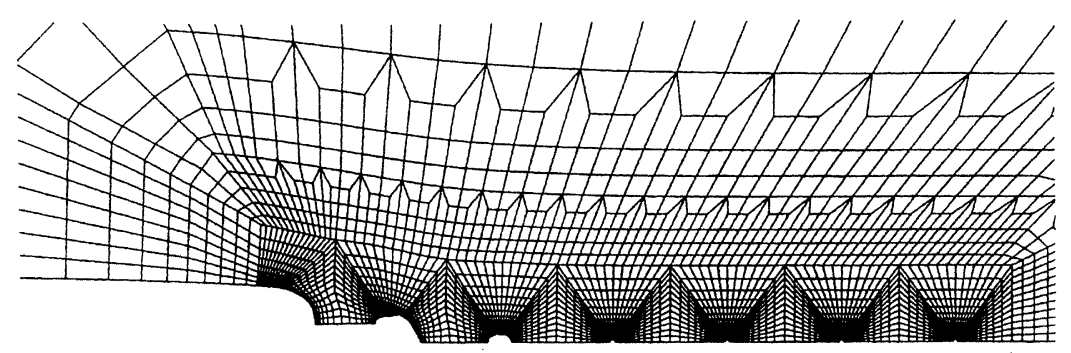

(b)

Fig. 8. (a) Deformed mesh following failure of the fourth ligament for $f_{0}=0.014$ and (b) deformed mesh following failure of the second ligament for $f_{0}=0.00087$ showing clear evidence of the void by void growth mechanism. 
void volume fraction considered in the present plane strain model, $f_{0}=0.00087$, is representative of tough alloys with very high tearing resistance. For such materials, the definition of $J_{\text {Ic }}$ has always been problematic because of the lack of a precise event characterizing the initiation of crack growth. The ASTM (1997) standard proposes that $J_{\text {Ic }}$ be taken as the value of $J$ at $\Delta a=0.2 \mathrm{~mm}$. In the numerical modeling in this paper, $J_{\text {Ic }}$ is identified as $J$ at failure of the first ligament, which is effectively when $\Delta a=X_{0}$. Values of $X_{0}$ in the range from 0.1 to $0.2 \mathrm{~mm}$ are not uncommon for the primary population of void nucleating particles in some tough pressure vessel steels (Gao et al., 1998). Thus, for these materials the present definition is not too different from the ASTM proposal. However, for materials with large $S$ (i.e. sufficiently small $f_{0}$ ) but $X_{0}$ much smaller than $0.2 \mathrm{~mm}$, the criterion for initiation employed here will obviously underestimate $J_{\text {Ic }}$ as defined by the ASTM proposal. For such materials, according to the ASTM proposal, initiation of growth takes place after the tip has advanced past multiple voids.

\section{Relationship of present model to a model with an embedded fracture process zone}

In this section, a quantitative connection is made between the present two-dimensional, discrete void model and the earlier embedded fracture process model of ductile crack initiation and growth of Tvergaard and Hutchinson (1992). Specific properties of the cohesive separation law used to represent the fracture process in the earlier model were derived from a three-dimensional model of interacting voids. These results will be compared with results computed for the present two-dimensional geometry for the purpose of correlating corresponding values of $f_{0}$. In addition, the present results for crack growth resistance will be related to the predictions of the earlier model, as will the results for the transition from the multiple to single void mechanisms.

Traction-separation curves representing the ductile fracture process are presented in Fig. 9 for uniform planar arrays of two- and three-dimensional void geometries, in each case for the material properties

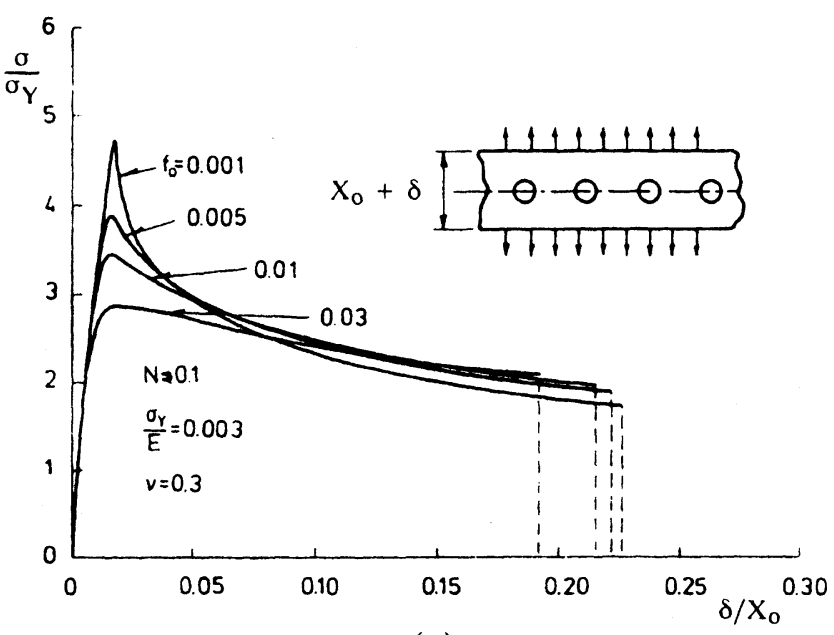

(a)

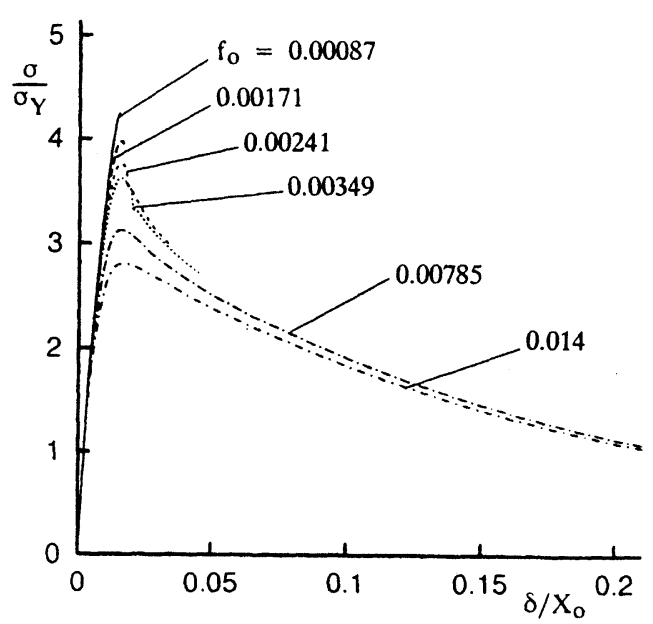

(b)

Fig. 9. Traction-separation curves for the multiple void mechanism as computed for an infinite planar slab of initial height $X_{0}$ subject to uniform normal separation of the top and bottom faces: (a) for initially spherical voids as modeled by the Gurson model under conditions of uniaxial straining (Tvergaard and Hutchinson, 1992) and (b) for two-dimensional cylindrical voids as computed using the present finite strain, plane strain formulation. Definitions of the initial void volume fractions are given in the text. For both cases, $N=0.1, \sigma_{\mathrm{Y}} / E=0.003$ and $v=0.3$. 
specified in (6). The initial height of the slab containing the plane of voids is taken to be the same as the spacing between the voids, $X_{0}$. The top and bottom of the slab undergo a uniform relative normal displacement, $\delta$, with zero tangential displacement such that all the voids undergo the same deformation history. The curves for the three-dimensional initially spherical voids in Fig. 9a were computed using the Gurson (1977) model under conditions of uniaxial straining, as described in Tvergaard and Hutchinson (1992). The centers of the voids are imagined to be in a doubly periodic array lying in the center plane of the slab. The initial void volume fraction, $f_{0}$, is taken to be the same as that used in the Gurson model: with $R_{0}$ as the radius of the initial spherical void and the outer surface of the spherical shell in the model taken as $X_{0} / 2$, the interpretation of $f_{0}$ would be $\left(f_{0}\right)_{3 \mathrm{D}}=\left(2 R_{0} / X_{0}\right)^{3}$. The curves for the two-dimensional, initially cylindrical voids were computed using the same finite strain, finite element formulation used in the present study but applied to a unit periodic cell of dimension $X_{0}$ by $X_{0}$ subject to plane strain and overall uniaxial staining. In this case, the initial volume fraction is the same at that used in the previous sections: with $R_{0}$ as the initial radius of the cylindrical void, $\left(f_{0}\right)_{2 \mathrm{D}}=\pi\left(R_{0} / X_{0}\right)^{2}$.

The traction-separation curves in two- and three-dimensions in Fig. 9 are reasonably close at similar values of $f_{0}$. This suggests that the present two-dimensional plane strain model of crack growth should provide a reasonable approximation to three-dimensional models that would be far more computationally intensive. ${ }^{1}$ The two fracture process parameters of most importance are the work of separation per unit area, $\Gamma_{0}=\int \sigma \mathrm{d} \delta$, and the peak separation stress, $\hat{\sigma}$. The work of separation depends somewhat on the point at which localization of deformation in the ligaments between the voids sets in. Localization sets in when the void volume fraction reaches a level of approximately $f=0.2$, corresponding to $\delta / X_{0} \cong 0.2$. $^{2}$ The work of separation (i.e. the area under a given curve in Fig. 9) is approximately

$$
\Gamma_{0} \cong 0.5 \sigma_{\mathrm{Y}} X_{0}
$$

for both the two- and three-dimensional models with only a weak dependence on $f_{0}$. The dependence of the peak stress on $f_{0}$ is highly significant, however, since the peak stress has an exceptional effect on the total toughness (see below). The normalized peak stress is displayed for each of the two models in Fig. 10. The estimate from the two-dimensional model lies below that from the three-dimensional model, but the difference is not great. Further details for the three-dimensional model, including the influences of $N$ and $\sigma_{\mathrm{Y}} / E$ on $\Gamma_{0}$ and $\hat{\sigma}$, are given by Tvergaard and Hutchinson (1992).

In the regime in which the multiple void mechanism is operative, the traction-separation relation derived from the above slab model can be used to model a cohesive failure plane ahead of a crack tip. In this spirit, a traction-separation law characterized by $\Gamma_{0}$ and $\hat{\sigma}$ is embedded as a cohesive zone within an elasticplastic continuum. Tvergaard and Hutchinson (1992) computed resistance curves for plane strain mode I crack growth in small scale yielding. According to the embedded process zone model, initiation of growth occurs when the remote $J$ attains the work of the fracture process, i.e.

$$
J_{\mathrm{Ic}}=\Gamma_{0}
$$

The resistance curve, $J_{\mathrm{R}}(\Delta a)$, approaches a steady-state value $\Gamma_{\mathrm{SS}}$ as the crack extends to distances that are on the order of the plastic zone size. The curve of $\Gamma_{\mathrm{SS}} / \Gamma_{0}$ as a function of $\hat{\sigma} / \sigma_{\mathrm{Y}}$ as computed from the embedded cohesive zone model is presented in Fig. 11a for the material properties in (6). As discussed more fully by Tvergaard and Hutchinson (1992), values of $\hat{\sigma} / \sigma_{\mathrm{Y}}$ above about 3 give rise to a substantial plastic

\footnotetext{
${ }^{1}$ In passing, it can be noted that the correspondence between the two- and three-dimensional results in Fig. 9 can be improved if the initial void volume fraction in the three-dimensional model uses the volume of a cube of dimension $X_{0}$ as the reference volume per unit cell rather than a sphere of radius $X_{0} / 2$. Then, $f_{0}=(4 \pi / 3)\left(R_{0} / X_{0}\right)^{3}$, which reduces the initial volume fraction associated with each of the curves in Fig. 9 a by a factor $\pi / 6$.

${ }^{2}$ See Pardoen and Hutchinson (2000) for a discussion of more refined criteria for the final localization in the ligaments.
} 


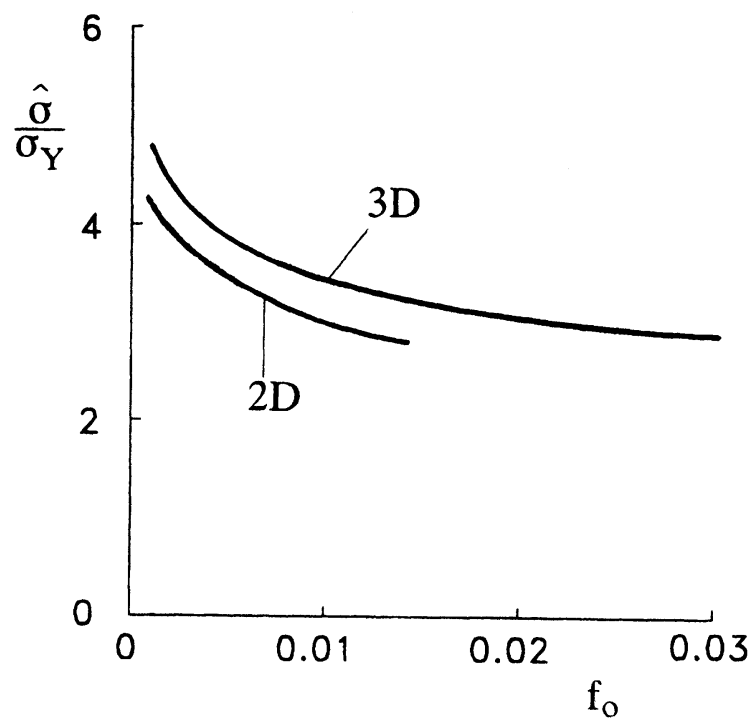

Fig. 10. Dependence of the peak separation stress, $\hat{\sigma}$, in the multiple void mechanism as determined from the results in Fig. 9 on $f_{0}$ for the two- and three-dimensional models. Definitions of the initial void volume fractions are given in the text. For both cases, $N=0.1$, $\sigma_{\mathrm{Y}} / E=0.003$ and $v=0.3$.
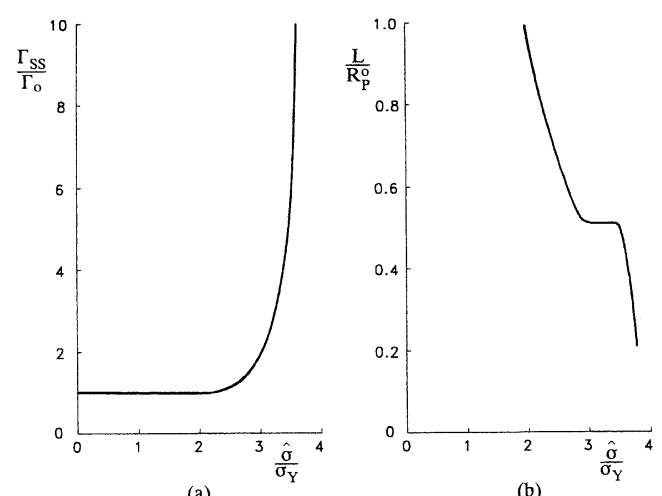

Fig. 11. (a) Steady-state toughness as predicted by a cohesive zone model based on the multiple void mechanism as a function of normalized peak separation stress and (b) the computed length of the fracture process zone as a function of the peak separation stress. For $N=0.1, \sigma_{\mathrm{Y}} / E=0.003$ and $v=0.3$ (Tvergaard and Hutchinson, 1992).

zone surrounding the cohesive zone causing significant additional irreversible dissipation and providing a large amplification of the work of the fracture process.

The computed length $L$ of the cohesive fracture process zone is plotted as a function of $\hat{\sigma} / \sigma_{\mathrm{Y}}$ in Fig. $11 \mathrm{~b}$. This length is normalized by

$$
R_{0}=\frac{1}{3 \pi\left(1-v^{2}\right)} \frac{E \Gamma_{0}}{\sigma_{\mathrm{Y}}^{2}}
$$

which, by (9), is the standard estimate of the half height of the plastic zone at the initiation of crack growth in small scale yielding, i.e. $R_{\mathrm{P}} \cong(1 / 3 \pi)\left(K_{\mathrm{Ic}} / \sigma_{\mathrm{Y}}\right)^{2}$. By $(8), R_{0}$ can be rewritten in terms of the spacing between voids, $X_{0}$, as 


$$
R_{0}=0.053 \frac{E}{\left(1-v^{2}\right) \sigma_{\mathrm{Y}}} X_{0}
$$

For $\sigma_{\mathrm{Y}} / E=0.003, R_{0} \cong 18 X_{0}$. Inspection of Fig. $11 \mathrm{~b}$ reveals that, indeed, the length of the fracture process zone is many times the void spacing when $\hat{\sigma} / \sigma_{\mathrm{Y}}<4$, consistent with the assumption of a multiple void mechanism. However, as $\hat{\sigma} / \sigma_{\mathrm{Y}}$ approaches $4, L$ plummets and at some point the embedded process zone model predicts that the cohesive zone will become on the order of $X_{0}$. The embedded process zone based on the multiple void mechanism is then no longer valid, and the transition towards the single void mechanism occurs.

The predictions for the transition from the multiple to single void mechanisms from the cohesive zone model are similar to those from the two-dimensional discrete void model analyzed in Section 3 . The cohesive zone model based on the Gurson model estimates of the fracture process parameters predicts the transition to occur at $f_{0} \cong 0.005$, while the present discrete void calculation of Section 3 gives the slightly lower estimate as $f_{0} \cong 0.003$. It must be emphasized that these values have been computed for one specific value of the strain hardening exponent, $N=0.1$, and it is expected that the transition will depend to some extent on $N$. It is also worth noting that the estimate of $J_{\text {Ic }}$ from the cohesive zone model from (8) and (9), $J_{\text {Ic }} \cong 0.5 \sigma_{\mathrm{Y}} X_{0}$, agrees reasonably closely with the values computed for the discrete void model when the initial void volume fraction is such that the multiple void mechanism applies.

\section{Conclusions}

The discrete void model analyzed in this paper clearly reveals two distinct limiting mechanisms of ductile crack growth - growth by multiple void interaction and void by void growth. The underlying mechanism is the same in each limit, i.e. void nucleation, growth and coalescence, and in this sense there is really only one basic mechanism. However, the distinction between the two limits is useful in constructing models of the fracture process. The two types of models employed in the past (single void/crack interaction and cohesive zone modeling of multiple voids) are quite different, yet both effective in their respective regimes. The transition between the two mechanisms is primarily governed by the initial void volume fraction, $f_{0}$. The best estimate of the transition that can be inferred from the present calculations is that it occurs for $f_{0}$ in a fairly broad range from about 0.001 to 0.005 . This level of $f_{0}$ is thought to be representative of initial void populations (or populations of void nucleating particles) of many structural alloys. As a practical matter, the importance of recognizing the existence of the two mechanisms and the transition between them lies in the fact that the toughness implications of the two models differ by more than a factor of two. Equation (1) applies to both models, but $C_{\mathrm{SV}}$ is two or more times $C_{\mathrm{MV}}$. The discrete model analyzed here (c.f. Fig. 2) brings out the transition between $C_{\mathrm{MV}}$ and $C_{\mathrm{SV}}$ as $f_{0}$ decreases. In the transition between the two mechanisms, where many structural alloys would appear to lie, processing changes to diminish $f_{0}$ will have a significantly larger influence on increasing toughness than either of the limiting models would predict.

While highly idealized, the present model provides insight into earlier attempts to correlate experimentally measured initiation toughness with micro-structural data on initial void populations and to relate these to the predictions of single void models. Fig. 12 is derived from Fig. 12 in the paper by McMeeking (1977). As in the original plot, the experimental points have plotted as the crack tip opening displacement at initiation normalized by void spacing, $\delta_{\mathrm{t}} / X_{0}$, against the ratio of the particle spacing to the size of the void nucleating particles, $X_{0} /\left(2 R_{0}\right)$. The early prediction for the single void model from Rice and Johnson (1970) and McMeeking (1977) is included in this figure. As already emphasized, this prediction significantly overestimates toughness for pre-existing voids due to the neglection of interaction effects. In small scale yielding in plane strain, $\delta_{\mathrm{t}} / X_{0} \cong 0.5 J_{\mathrm{Ic}} /\left(\sigma_{\mathrm{Y}} X_{0}\right)=0.5 C$ where $C$ is the dimensionless factor defined in (1). The scale for $C$ is superimposed on Fig. 12. In three dimensions, the ratio, $X_{0} /\left(2 R_{0}\right)$, is also approximately 


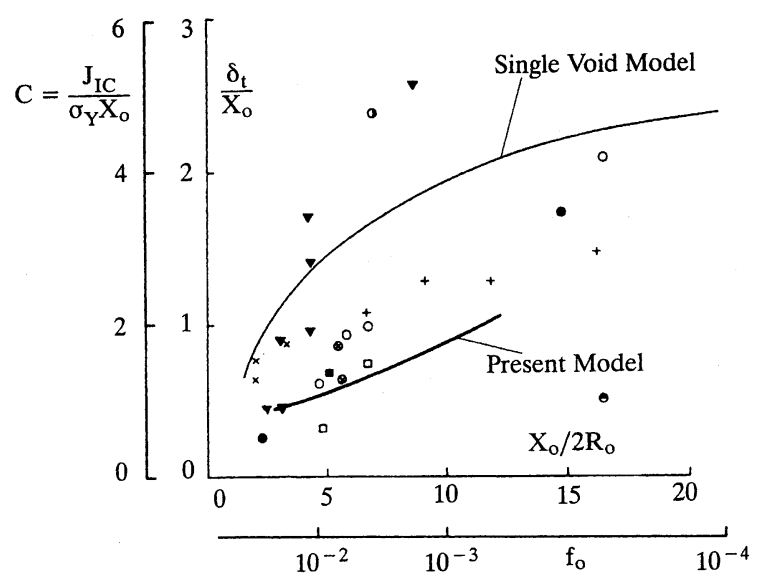

Fig. 12. Original experimental data of critical crack tip opening displacement, $\delta_{\mathrm{t}} / X_{0}$ versus dimensionless void nucleating particle characteristic, $X_{0} /\left(2 R_{0}\right)$, assembled by McMeeking (1977), including the upper curve as the theoretical prediction for model of Rice and Johnson (1970) based on the single void mechanism. As discussed in the text, a new ordinate, $C \equiv J_{\mathrm{Ic}} /\left(\sigma_{\mathrm{Y}} X_{0}\right)=2 \delta_{\mathrm{t}} / X_{0}$, and a new abscissa, $f_{0}=\left(2 R_{0} / X_{0}\right)^{3}$, have been superimposed on the figure. The prediction from the present two-dimensional discrete void model is included (the result from Fig. 2 with $\chi_{\mathrm{c}}=0.3, r_{0} / X_{0}=0.1, N=0.1, \sigma_{\mathrm{Y}} / E=0.003$ and $v=0.3$ ).

$\left(1 / f_{0}\right)^{1 / 3}$, and a scale for $f_{0}$ is superimposed on the abscissa in the same figure. The relation between $C$ and $f_{0}$ from Fig. 2 as determined by the present model (with $r_{0}=0.1, \chi_{\mathrm{c}}=1 / 2$ and $N=0.1$ ) is also plotted in Fig. 12. The present result approaches the single void model result of Aravas and McMeeking (1985) $\left(C \cong 1.1\right.$ for $N=0.1$ and $f_{0} \cong 10^{-3}$ ) when the single void mechanism pertains at small $f_{0}$ and drops below those results at larger $f_{0}$. At $f_{0}=10^{-2}$, the present result is about one half that predicted by Aravas and McMeeking (1985). Thus, the present results are seen to bridge the limiting results from single and multiple void models.

With the exception of two of the experimental points, the curve from the present study passes through, or lies below, most of the data. In addition to the intrinsic difficulty of measuring and assigning values to $X_{0} /\left(2 R_{0}\right)$ for actual materials, the comparison of theory and experiment in Fig. 12 is complicated by void nucleation effects that can substantially increase toughness when nucleation is delayed. Void shape and distribution effects have not been taken into account, nor has there been any attempt to match hardening of the test materials with that in the models. Nevertheless, the present model does give reasonable agreement with experimental toughness measurements for materials whose void nucleating particles have relatively large initial void volume fractions. The present work suggests that some of the discrepancy between the data and the single void model predictions in this range is due to the inapplicability of this model. Otherwise, the significant overestimate of toughness would be difficult to understand since the model results were derived under the assumption that voids nucleate at zero strain and thus would be expected to underestimate toughness initiation.

While a model based on discrete voids such as the one employed here has been highly successful at revealing basic aspects of the mechanism of crack growth, its computational complexity renders it impractical for engineering crack growth studies. This would certainly be the true if one were to attempt to analyze more realistic three-dimensional void populations or even of two-dimensional void populations where the voids did not all lie on the fracture plane. We believe that an intermediate approach based on void-containing finite elements offers a practical alternative that should be able to capture most of the behaviors exposed here. These are the finite element approaches based on dilatational plasticity models, such as that of Gurson (1977), which rely on calibration using experimental crack growth data for a given material to determine micro-structural parameters such as $X_{0}$ and $f_{0}$. The extensive literature on this class of 
models, alluded to earlier, presents a convincing case for its utility in engineering analysis. As emphasized in Section 1, these models have not yet been used to any extent to explore trends in toughness and crack growth resistance as related to changes in micro-structure as reflected in the population of initial voids or void-nucleating particles. Recent extensions of the Gurson model to include void shape and distribution effects (Pardoen and Hutchinson, 2000) open up opportunities in this direction. There continues to be a role for discrete void models in more fundamental studies of the present type, at the very least to help calibrate and assess the engineering models.

A final issue related to potential void size effects should be raised. The present study of ductile fracture, like nearly all others that have proceeded it, has employed conventional plasticity theory. By conventional, one means a theory of plasticity that has been developed and calibrated for applications at macroscopic scales greater than the micron scale. Recent studies (Fleck and Hutchinson, 1997) have shown that significant hardening enhancements arise when non-uniform plastic deformation takes place at the micron scale. Tests on wire torsion, sheet bending, and indentation hardness all display effective strength increases of factors of two to three when the controlling dimension is measured in microns. It seems highly unlikely that void growth should escape these size effects, and theoretical studies of void growth based on micron scale plasticity (Fleck and Hutchinson, 1997) confirm this. In practical terms, this means that voids smaller than a certain characteristic size will experience increased resistance to growth. Firm estimates of this characteristic size are not yet available, but it appears likely to be on the order of $1 \mu \mathrm{m}$. If materials could be processed such that the initial voids or void-nucleating particles fell below this characterize size, toughness enhancements would ensue over and above those predicted by the various models cited in this paper, all of which are based on conventional plasticity.

\section{Acknowledgements}

The authors acknowledge insightful discussions with T. Pardoen of the Département des Sciences des Matériaux et des Procédés, Université catholique de Louvain. The work of JWH is supported in part by grants NSF CMS-96-34632 and AFSOR SA1542-22500 PG, and by the Division of Engineering and Applied Sciences. JWH would also like to acknowledge support from the Danish Engineering Foundation during his stay in Denmark.

\section{References}

ASTM, 1997. Annual book of standards. Standard E1820-96: standard test method for measurement of fracture toughness. ASTM, Philadelphia, PA.

Aravas, N., McMeeking, R.M., 1985. Finite element analysis of void growth near a blunting crack tip. J. Mech. Phys. Solids 33, 25-49.

Brocks, W.K., Klingbeil, Kunecke, D., Sun, D.-Z., 1995. Application of the Gurson model to ductile tearing resistance. In: Kirk, M., Bakker, A. (Eds.), Constraint Effects in Fracture: Theory and Applications. ASTM STP 1244, Philadelphia, PA.

Budiansky, B., Hutchinson, J.W., Slutsky, S., 1982. Void growth and collapse in viscous solids. In: Hopkins, H.G., Sewell, M.J. (Eds.), Mechanics of Solids. Pergamon Press, Oxford, pp. 13-46.

Eshelby, J.D., 1970. The energy momentum tensor in continuum mechanics. In: Kanninen, M.F. et al. (Eds.), Inelastic Behavior of Solids. McGraw-Hill, New York.

Fleck, N.A., Hutchinson, J.W., 1997. Strain gradient plasticity. Adv. Appl. Mech. 23, 295-361.

Gao, X., Faleskog, J., Shih, C.F., Dodds, R.H., 1998. Ductile tearing in part-through cracks: experiments and cell-model predictions. Engng. Frac. Mech. 59, 761-777.

Gurson, A.L., 1977. Continuum theory of ductile rupture by void nucleation and growth: Part I: Yield criteria and flow rules for porous ductile media. J. Engng. Mater. Technol. 99, 2-15.

Hom, C.L., McMeeking, R.M., 1989. Three-dimensional void growth before a blunting crack tip. J. Mech. Phys. Solids $37,395-415$. 
McMeeking, R.M., 1977. Finite deformation analysis of crack-tip opening in elastic-plastic materials and implications for fracture. J. Mech. Phys. Solids 25, 357-381.

Needleman, A., Tvergaard, V., 1987. An analysis of ductile rupture modes at a crack tip. J. Mech. Phys. Solids 35, 151-183.

Pardoen, T., Hutchinson, J.W., 2000. An extended model for void growth and coalescence. J. Mech. Phys. Solids 48, $2467-2512$.

Rice, J.R., 1968. A path independent integral and the approximate analysis of strain concentration by notches and cracks. J. Appl. Mech. 90, 379-386.

Rice, J.R., Johnson, M.A., 1970. In: Kanninen, M.F. et al. (Eds.), Inelastic Behavior of Solids. McGraw-Hill, New York.

Rousselier, G., 1987. Ductile fracture models and their potential in local approach of fracture. Nucl. Engng. Des. 105, 97-111.

Tvergaard, V., 1997. Studies of void growth in a thin ductile layer between ceramics. Computat. Mech. 20, 186-191.

Tvergaard, V., Hutchinson, J.W., 1992. The relation between crack growth resistance and fracture process parameters in elastic-plastic solids. J. Mech. Phys. Solids 40, 1377-1397. 